\title{
The Ionization Problem
}

\author{
Phan Thành Nam (Ludwig-Maximilians-Universität München, Germany)
}

The question: "How many electrons can a nucleus bind?" is as old as quantum mechanics, but its rigorous answer based on the many-body Schrödinger equation remains a difficult challenge to mathematicians. Nevertheless, there has been remarkable progress in this problem in the past four decades. We will review the current understanding of the Schrödinger equation and then turn to simplified models where the problem has been solved satisfactorily. We will also discuss the connection to the liquid drop model, which is somewhat more classical, but no less interesting.

\section{Atomic Schrödinger equation}

For us, an atom is a system of $N$ quantum electrons of charge -1 moving around a heavy classical nucleus of charge $Z \in$ $\mathbb{N}$ and interacting via Coulomb force (we use atomic units). The wave functions of $N$ electrons are normalised functions in $L^{2}\left(\mathbb{R}^{3 N}\right)$ satisfying the anti-symmetry

$$
\begin{aligned}
\Psi\left(x_{1}, \ldots, x_{i}, \ldots, x_{j}, \ldots, x_{N}\right) & \\
& =-\Psi\left(x_{1}, \ldots, x_{j}, \ldots, x_{i}, \ldots, x_{N}\right), \quad \forall i \neq j
\end{aligned}
$$

where $x_{i} \in \mathbb{R}^{3}$ stands for the position of the $i$-th electron (we will ignore the spin for simplicity). The Hamiltonian of the system is

$$
H_{N}=\sum_{i=1}^{N}\left(-\frac{1}{2} \Delta_{x_{i}}-\frac{Z}{\left|x_{i}\right|}\right)+\sum_{1 \leqslant i<j \leqslant N} \frac{1}{\left|x_{i}-x_{j}\right|} .
$$

The self-adjointness of $H_{N}$ follows a famous theorem of Kato. We are interested in the ground state problem

$$
E_{N}=\inf _{\|\Psi\|_{L^{2}}=1}\left\langle\Psi, H_{N} \Psi\right\rangle \text {. }
$$

By a standard variational method, we know that the minimizers, if they exist, are solutions to the Schrödinger equation

$$
H_{N} \Psi=E_{N} \Psi \text {. }
$$

The existence/nonexistence issue is related to the stability of the system, namely whether all electrons will be bound, or some of them may escape to infinity. Obviously, $H_{N}$ and $E_{N}$ also depend on $Z$, but let us not include this dependence in the notation.

It is natural to guess that there is a sharp transition when $N$ crosses the value $Z+1$. Heuristically, if $N<Z+1$, then the outermost electron sees the rest of the system as a large nucleus of the effective charge $Z-(N-1)>0$. Hence, this electron will "prefer to stay" by the Coulomb attraction. On the other hand, if $N>Z+1$, then the outermost electron will "prefer to go away" by the Coulomb repulsion.

Part of the above heuristic guess was justified by Zhislin in 1960 . 
Theorem 1. If $N<Z+1$, then $E_{N}$ has a minimizer.

More precisely, he proved that if $N<Z+1$, then $E_{N}<E_{N-1}$. This strict binding inequality prevents any electron from escaping to infinity, thus ensuring the compactness of minimising sequences for $E_{N}$. On a more abstract level, if $N<Z+1$, then $E_{N}$ is strictly below the essential spectrum of $H_{N}$. In fact, the essential spectrum of $H_{N}$ is $\left[E_{N-1}, \infty\right)$ due to the celebrated Hunziker-van Winter-Zhislin (HVZ) theorem.

Thus, Zhislin's theorem ensures the existence of all positive ions and neutral atoms. On the other hand, the nonexistence of highly negative ions is much more difficult, and often referred to as the "ionization conjecture"; see, e.g., [23, Problem 9] and [15, Chapter 12].

Conjecture 2. There exists a universal constant $C>0$ (possibly $C=1)$ such that if $N>Z+C$, then $E_{N}$ has no minimizer.

Note that the above heuristical argument is purely classical and it is too rough to understand the delicate quantum problem at hand. In 1983, Benguria and Lieb [1] proved that if the anti-symmetry condition of the wave functions is ignored, then the atoms with "bosonic electrons" always exist as soon as $N \leqslant t_{c} Z$ with a universal constant $t_{c}>1$ (numerically $t_{c} \approx 1.21$, computed by Baumgartner). Thus the ionization problem requires a deep insight, as the particle statistics, more precisely Pauli's exclusion principle, play an essential role.

\section{Known results}

A rigorous upper bound to the question "How many electrons can a nucleus bind?" was first derived by Ruskai [19] and Sigal [21] independently in 1982. They proved that there exists a critical value $N_{c}(Z)<\infty$ such that if $N>N_{c}(Z)$, then $E_{N}$ has no minimizer. In these works, they applied certain inequalities on classical point particles to the quantum problem via the geometric localization method. In particular, Sigal realized that for every collection $\left\{x_{i}\right\}_{i=1}^{N} \subset \mathbb{R}^{3}$ with $N>2 Z+1$, the energy contributed by the farthest electron, $x_{N}$ says, is always positive because of the triangle inequality

$$
-\frac{Z}{\left|x_{N}\right|}+\sum_{i=1}^{N-1} \frac{1}{\left|x_{i}-x_{N}\right|} \geqslant-\frac{Z}{\left|x_{N}\right|}+\frac{N-1}{2\left|x_{N}\right|}>0 .
$$

This leads to the upper bound $\lim \sup _{Z \rightarrow \infty} N_{c}(Z) / Z \leqslant 2$ in [22].

Later, Lieb, Sigal, Simon and Thirring [13] found the following improvement: for every $\left\{x_{i}\right\}_{i=1}^{N} \subset \mathbb{R}^{3}$ with $N$ large, one has

$$
\max _{1 \leqslant j \leqslant N}\left\{\sum_{1 \leqslant i \leqslant N, i \neq j} \frac{1}{\left|x_{i}-x_{j}\right|}-\frac{N+o(N)}{\left|x_{j}\right|}\right\} \geqslant 0 .
$$

Consequently, they obtained the asymptotic neutrality

$$
\lim _{Z \rightarrow \infty} \frac{N_{c}(Z)}{Z}=1 \text {. }
$$

It is unclear whether one can improve the quantity $N+o(N)$ in (1) to $N+O\left(N^{\alpha}\right)$ with some constant $0 \leqslant \alpha<1$.

In 1990, Fefferman and Seco [4], and Seco, Sigal and Solovej [20], proved

Theorem 3. When $Z \rightarrow \infty$, we have $N_{c}(Z) \leqslant Z+O\left(Z^{5 / 7}\right)$.
This bound was obtained by comparing it with the Thomas-Fermi theory (that we will revisit below) and taking into account quantitative estimates for Scott's correction (studied by Huges, and by Siedentop and Weikard). There has been no further improvement in the past three decades!

Instead of the asymptotics as $Z \rightarrow \infty$, one may also be interested in explicit bounds for all $Z$ (in fact, $1 \leqslant Z \leqslant 118$ for realistic atoms in the current periodic table). The best known result in this direction is

Theorem 4 ([12, 17]). For all $Z \geqslant 1, N_{c}(Z)<\min (2 Z+$ $\left.1,1.22 Z+3 Z^{1 / 3}\right)$.

Let us quickly explain Lieb's proof of the bound $2 Z+1$ in [12], since it is short and important. The starting point is the following identity, which follows from the Schrödinger equation

$$
\left\langle\left|x_{N}\right| \Psi_{N},\left(H_{N}-E_{N}\right) \Psi_{N}\right\rangle=0 .
$$

The idea of "multiplying the equation by $|x|$ " was also used by Benguria on a simplified model. Then we decompose

$$
H_{N}=H_{N-1}-\Delta_{N}+\sum_{i=1}^{N-1} \frac{1}{\left|x_{i}-x_{N}\right|} .
$$

For the first $(N-1)$ electrons, we use the obvious inequality

$$
H_{N-1} \geqslant E_{N-1} \geqslant E_{N} \text {. }
$$

For the $N$-th electron, we use the operator inequality

$$
(-\Delta)|x|+|x|(-\Delta) \geqslant 0 \text { on } L^{2}\left(\mathbb{R}^{3}\right)
$$

(which is equivalent to Hardy's inequality). Consequently,

$$
Z>\sum_{i=1}^{N}\left\langle\Psi, \frac{\left|x_{N}\right|}{\left|x_{i}-x_{N}\right|} \Psi\right\rangle=\frac{1}{2} \sum_{i=1}^{N}\left\langle\Psi, \frac{\left|x_{N}\right|+\left|x_{i}\right|}{\left|x_{i}-x_{N}\right|} \Psi\right\rangle>\frac{N-1}{2}
$$

Thus $N<2 Z+1$. Here, we have used the symmetry of $|\Psi|^{2}$ and the triangle inequality.

To get the bound in [17], we multiply Schrödinger's equation with $\left|x_{N}\right|^{2}$ instead of $\left|x_{N}\right|$ and proceed similarly. In this case, the operator $(-\Delta)|x|^{2}+|x|^{2}(-\Delta)$ on $L^{2}\left(\mathbb{R}^{3}\right)$ is not positive, but its negative part can be controlled using a special property of the ground state. The key point is, instead of using the triangle inequality, we now have

$$
Z \geqslant \inf _{\left\{x_{i}\right\}_{i=1}^{N} \subset \mathbb{R}^{3}} \frac{\sum_{1 \leqslant i<j \leqslant N} \frac{\left|x_{i}\right|^{2}+\left|x_{j}\right|^{2}}{\left|x_{i}-x_{j}\right|}}{(N-1) \sum_{i=1}^{N}\left|x_{i}\right|}+O\left(N^{2 / 3}\right)=\beta N+O\left(N^{2 / 3}\right)
$$

with the statistical value

$$
\beta:=\inf _{\substack{\rho \text { probability } \\ \text { measure in } \mathbb{R}^{3}}}\left\{\frac{\iint_{\mathbb{R}^{3} \times \mathbb{R}^{3}} \frac{x^{2}+y^{2}}{2|x-y|} \mathrm{d} \rho(x) \mathrm{d} \rho(y)}{\int|x| \mathrm{d} \rho(x)}\right\} .
$$

It is nontrivial to compute $\beta$, but we can estimate it using the inequality

$$
\begin{aligned}
\iint_{\mathbb{R}^{3} \times \mathbb{R}^{3}} \frac{x^{2}+y^{2}}{|x-y|} \mathrm{d} \rho(x) \mathrm{d} \rho(y) \\
\quad \geqslant \iint_{\mathbb{R}^{3} \times \mathbb{R}^{3}}\left(\max (|x|,|y|)+\frac{\min (|x|,|y|)^{2}}{|x-y|}\right) \mathrm{d} \rho(x) \mathrm{d} \rho(y) .
\end{aligned}
$$

which is a consequence of (1). This gives $\beta \geqslant 0.82$, leading to the bound $1.22 Z+3 Z^{1 / 3}$ (as $\beta^{-1} \leqslant 1.22$ ). 


\section{Thomas-Fermi theory}

Since the Schrödinger equation is too complicated, for practical computations one often relies on approximate models which are nonlinear but dependent on less variables. In density functional theory, a popular method in computational physics and chemistry, one replaces the $N$-body wave function $\Psi$ with its one-body density

$$
\rho_{\Psi}(x)=N \int_{\mathbb{R}^{3(N-1)}}\left|\Psi\left(x, x_{2}, \ldots, x_{N}\right)\right|^{2} d x_{2} \ldots d x_{N} .
$$

Clearly, $\rho_{\Psi}: \mathbb{R}^{3} \rightarrow[0, \infty)$ and $\int_{\mathbb{R}^{3}} \rho_{\Psi}=N$.

The oldest density functional theory was proposed by Thomas and Fermi in 1927. In the Thomas-Fermi (TF) theory, the ground state energy $E_{N}$ is replaced by its semiclassical approximation

$$
\begin{aligned}
E^{\mathrm{TF}}(N)=\inf _{\int \rho=N}\left\{C^{\mathrm{TF}} \int_{\mathbb{R}^{3}}\left(\rho^{5 / 3}(x)-\frac{Z}{|x|} \rho(x)\right) d x\right. \\
\left.+\frac{1}{2} \int_{\mathbb{R}^{3}} \int_{\mathbb{R}^{3}} \frac{\rho(x) \rho(y)}{|x-y|} d x d y\right\}
\end{aligned}
$$

with a constant $C^{\mathrm{TF}}>0$. The existence and properties of the TF minimizers was studied by Lieb and Simon in [14]. In particular, they proved

Theorem 5. $E^{\mathrm{TF}}(N)$ has a minimizer if and only if $N \leqslant Z$.

By standard techniques, we find that the TF functional is convex and rotation invariant. Therefore, if a minimizer exists, it is unique and radial. Moreover, it satisfies the TF equation

$$
\frac{5}{3} C^{\mathrm{TF}} \rho(x)^{2 / 3}=\left[Z|x|^{-1}-\rho *|x|^{-1}-\mu\right]_{+}
$$

for some chemical potential $\mu \geqslant 0$.

The existence of the TF minimizer is rather similar to Zhislin's theorem for the Schrödinger equation. The nonexistence is more challenging. The original proof of Lieb and Simon is based on a clever use of the maximum principle. Here we offer another proof, using a variant of the BenguriaLieb argument.

Proof of $N \leqslant Z$ [18]. Assume that the TF equation has a radial solution $\rho$. Multiplying the equation with $|x|^{k} \rho(x), k \geqslant 1$, we have the pointwise inequality

$$
\left(Z|x|^{-1}-\rho *|x|^{-1}-\mu\right) \rho(x)|x|^{k}=\frac{5}{3} C^{\mathrm{TF}} \rho(x)^{5 / 3}|x|^{k} \geqslant 0 .
$$

Then we integrate over $\{|x| \leqslant R\}$. Note that $\mu \geqslant 0$. Moreover, since $\rho$ is radial, by Newton's theorem we have

$$
\rho *|x|^{-1}=\int_{\mathbb{R}^{3}} \frac{\rho(y)}{\max (|x|,|y|)} d y .
$$

Consequently,

$$
\begin{aligned}
Z \int_{|x| \leqslant R}|x|^{k-1} \rho(x) & \geqslant \int_{|x| \leqslant R}|x|^{k} \rho(x)\left(\rho *|x|^{-1}\right) d x \\
& \geqslant \frac{1}{2} \iint_{|x|,|y| \leqslant R} \frac{\left(|x|^{k}+|y|^{k}\right) \rho(x) \rho(y)}{\max (|x|,|y|)} d x d y .
\end{aligned}
$$

On the other hand, by the AM-GM inequality,

$$
\frac{|x|^{k}+|y|^{k}}{\max (|x|,|y|)} \geqslant\left(1-\frac{1}{k}\right)\left(|x|^{k-1}+|y|^{k-1}\right) \text {. }
$$

Thus

$$
\begin{aligned}
Z \int_{|x| \leqslant R}|x|^{k-1} \rho(x) d x & \\
& \geqslant\left(1-\frac{1}{k}\right)\left(\int_{|x| \leqslant R}|x|^{k-1} \rho(x) d x\right)\left(\int_{|y| \leqslant R} \rho(y) \mathrm{d} y\right) .
\end{aligned}
$$

Taking $R \rightarrow \infty$ and $k \rightarrow \infty$, we conclude that $\int_{\mathbb{R}^{3}} \rho \leqslant Z$.

When $N=Z$, the TF minimizer has the perfect scaling property:

$$
\rho_{Z}^{\mathrm{TF}}(x)=Z^{2} \rho_{1}^{\mathrm{TF}}\left(Z^{1 / 3} x\right), \quad \forall x \in \mathbb{R}^{3}
$$

where the function $\rho_{1}^{\mathrm{TF}}$ is independent of $Z$. Moreover, it satisfies the TF equation with chemical potential 0 . Thus if we denote the TF potential

$$
\varphi_{Z}^{\mathrm{TF}}(x)=Z|x|^{-1}-\rho_{Z}^{\mathrm{TF}} *|x|^{-1},
$$

then the TF equation can be written as the nonlinear Schrödinger equation

$$
\Delta \varphi_{Z}^{\mathrm{TF}}(x)=4 \pi\left(\frac{5}{3} C^{\mathrm{TF}}\right)^{-3 / 2} \varphi_{Z}^{\mathrm{TF}}(x)^{3 / 2} .
$$

This leads to the following Sommerfeld estimate [24, Theorem 4.6].

Theorem 6. Denote $A^{\mathrm{TF}}=\left(5 C^{\mathrm{TF}}\right)^{3}\left(3 \pi^{2}\right)^{-1}$ and $\zeta=(\sqrt{73}-$ 7)/2. Then

$$
A^{\mathrm{TF}} \geqslant \varphi_{Z}^{\mathrm{TF}}(x)|x|^{4} \geqslant A^{\mathrm{TF}}-C\left(Z^{1 / 3}|x|\right)^{-\zeta}, \quad \forall x \neq 0
$$

In particular, when $|x| \gg Z^{-1 / 3}$, then the TF potential $\varphi_{Z}^{\mathrm{TF}}$ is more or less independent of $Z$. This universality makes the TF approximation much more useful than what can normally be explained by its semiclassical nature. More precisely, the standard semiclassical analysis ensures that the TF theory gives a good approximation for the electron density in the distance $|x| \sim Z^{-1 / 3}$. However, we may expect that the TF theory gives a good approximation for larger distances, possibly up to $|x| \sim 1$. We refer to [25] for a detailed discussion.

\section{$4 \quad$ Hartree-Fock theory}

Invented shortly after the discovery of the Schrödinger equation, the Hartree-Fock (HF) theory has been a very useful computational method to describe electronic orbitals. In this theory, one restricts $N$-body wave functions to Slater determinants, or equivalently to their one-body density matrices which are trace class operators on $L^{2}\left(\mathbb{R}^{3}\right)$ satisfying

$$
0 \leqslant \gamma \leqslant 1, \quad \gamma=\gamma^{2}, \quad \operatorname{Tr} \gamma=N .
$$

The HF ground state energy is

$$
\begin{aligned}
E^{\mathrm{HF}}(N)=\inf _{\operatorname{Tr} \gamma=N}\left(\operatorname{Tr}\left(\left(-\Delta-Z|x|^{-1}\right) \gamma\right)\right. \\
\left.+\frac{1}{2} \iint_{\mathbb{R}^{3} \times \mathbb{R}^{3}} \frac{\rho_{\gamma}(x) \rho_{\gamma}(y)-|\gamma(x ; y)|^{2}}{|x-y|} d x d y\right)
\end{aligned}
$$

where $\rho_{\gamma}(x)=\gamma(x ; x)$ (the kernel of $\gamma$ is defined properly via the spectral decomposition).

The existence of Hartree-Fock minimizers when $N<Z+1$ was proved by Lieb and Simon in 1977. The nonexistence was proved later by Solovej in 2003 [24]. 
Theorem 7. There exists a universal constant $C>0$ such that if $N>Z+C$, then $E^{\mathrm{HF}}(N)$ has no minimizer.

To explain the proof, let us go back to the heuristic argument discussed before. Assume that we have an efficient method to separate $m$ outermost electrons. Then these particles see the rest of the system as a big nucleus with the effective nuclear charge $Z^{\prime}=Z-(N-m)$. Thus by the BenguriaLieb method, we may hope to get a bound like $m<2 Z^{\prime}+1$. Since $Z^{\prime}$ is smaller than $Z$, the loss of the factor 2 becomes less serious. If the procedure can be iterated to bring $Z^{\prime}$ down to order 1 , then we can conclude that $N-Z$ is of order 1 .

In [24], this approach is carried out rigorously by studying the screened nuclear potential

$$
\Phi_{Z}^{\mathrm{HF}}(x)=\frac{Z}{|x|}-\int_{|y| \leqslant|x|} \frac{\rho^{\mathrm{HF}}(y)}{|x-y|} d y .
$$

This function will be compared with the corresponding TF version

$$
\Phi_{Z}^{\mathrm{TF}}(x)=\frac{Z}{|x|}-\int_{|y| \leqslant|x|} \frac{\rho^{\mathrm{TF}}(y)}{|x-y|} d y .
$$

Similar to the TF potential $\varphi_{Z}^{\mathrm{TF}}(x), \Phi_{Z}^{\mathrm{TF}}(x)$ behaves as $|x|^{-4}$ for $|x| \gg Z^{-1 / 3}$. It turns out that this property holds true for the HF screened potential as well. The key ingredient of the analysis in [24] is

Theorem 8. There exist constants $C>0, \epsilon>0$ such that for all $x \neq 0$,

$$
\left|\Phi_{Z}^{\mathrm{HF}}(x)-\Phi_{Z}^{\mathrm{TF}}(x)\right| \leqslant C\left(1+|x|^{-4+\epsilon}\right) .
$$

This estimate can be proved by induction in $|x|$. First, for $|x| \leqslant Z^{-1 / 3+\epsilon}$, it follows by the semiclassical approximation. For longer distances, one repeatedly uses the Sommerfeld estimate (2) to get refined information for "inner electrons", and then controls the "outer electrons" in terms of the screened potential. At the end of the day, the universality of the TF potential makes a miracle happen!

Let us explain why Theorem 8 implies the ionization bound. First, Theorem 8 implies that for $|x|=r \sim 1$,

$$
\int_{|y| \leqslant r} \frac{\rho^{\mathrm{HF}}(y)-\rho^{\mathrm{TF}}(y)}{|x-y|} d y \leqslant C_{r} .
$$

We can replace $x$ by $v x$ with $v \in S^{2}$, then average over $v$ and use Newton's theorem. This gives

$$
Z^{\prime}:=\int_{|y| \leqslant r}\left(\rho^{\mathrm{HF}}(y)-\rho^{\mathrm{TF}}(y)\right) d y \leqslant C_{r} .
$$

The number of outermost electrons, namely $\int_{|y| \geqslant r} \rho^{\mathrm{HF}}$, can be controlled by a constant time $Z^{\prime}$, leading to the final bound $N-Z \leqslant C$.

Clearly, this proof strategy requires an efficient way of splitting the problem from the inside and the problem from the outside. This can be done for the Hartree-Fock theory, because the energy functional has been greatly simplified to a one-body functional. For the $N$-body Schrödinger equation, such a splitting would require a novel many-body localisation technique which is not available at the moment.

\section{$5 \quad$ Liquid drop model}

Now let us turn to a related problem in the liquid drop model which is somewhat more classical than the ionization conjec-

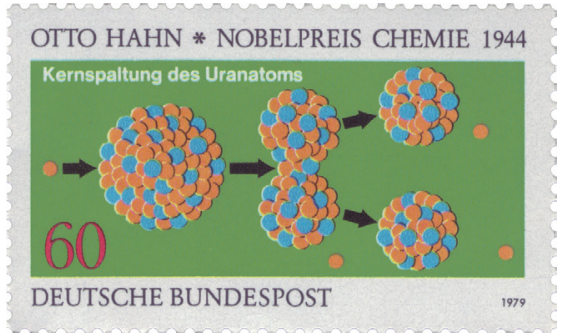

Figure 1. German stamp in 1979 honouring Otto Hahn (Wikipedia 2020)

ture. This model was proposed by Gamow in 1928 and further developed by Heisenberg, von Weizsäcker and Bohr in the 1930s. Recently, it has gained renewed interest from many mathematicians [3].

In modern language, a nucleus is described in this theory by an open set $\Omega \subset \mathbb{R}^{3}$ which solves the minimisation problem

$$
E^{\mathrm{G}}(m)=\inf _{|\Omega|=m}\left\{\operatorname{Per}(\Omega)+\frac{1}{2} \int_{\Omega} \int_{\Omega} \frac{1}{|x-y|} d x d y\right\} .
$$

Here $m$ stands for the number of nucleons (protons and neutrons) and $\operatorname{Per}(\Omega)$ is the perimeter in the sense of De Giorgi (which is the surface area of $\Omega$ when the boundary is smooth). The Coulomb term captures the electrostatic energy of protons.

It is generally assumed in physics literature that if a minimizer exists, then it is a ball. Consequently, by comparing the energy of a ball of volume $m$ with the energy of a union of two balls of volume $m / 2$, one expects the nonexistence of minimizers if $m>m_{*}$ with

$$
m_{*}=5 \frac{2-2^{2 / 3}}{2^{2 / 3}-1} \approx 3.518
$$

Conjecture 9 ([2]). If $m \leqslant m_{*}$, then $E^{\mathrm{G}}(m)$ is minimised by a ball. If $m>m_{*}$, then $E^{\mathrm{G}}(m)$ has no minimizer.

In particular, the nonexistence of minimizers for large $m$ is consistent with nuclear fission of heavy nuclei, which was discovered experimentally by Hahn and Strassmann in 1938.

Mathematically, it is nontrivial to analyse $E^{\mathrm{G}}(m)$ due to the energy competition: among all measurable sets of a given volume, a ball minimises the perimeter (by the isoperimetric inequality) but maximises the Coulomb self-interaction energy (by the Riesz rearrangement inequality).

In 2014, Knüpfer and Muratov [9] proved the following

Theorem 10. There exist constants $0<m_{1}<m_{2}$ such that:

(i) If $m<m_{1}$, then $E^{\mathrm{G}}(m)$ has a unique minimizer which is a ball;

(ii) If $m>m_{2}$, then $E^{\mathrm{G}}(m)$ has no minimizer.

The proof in [9] uses deep techniques in geometric measure theory, including a quantitative isoperimetric inequality proved by Fusco, Maggi and Pratelli in 2008. Independently, the existence of small $m$ was proved by Julin [10] and the nonexistence of large $m$ was proved by Lu and Otto [16]. In 2016, with Rupert Frank and Rowan Killip, we offered a new proof of the nonexistence which also provides the quantitative bound $m_{2} \leqslant 8$. Let us explain the short proofs in [10] and [5]. 
Proof. Existence for $m$ small [10]. Consider

$$
\begin{aligned}
& D(\Omega):=\operatorname{Per}(\Omega)+\frac{1}{2} \int_{\Omega} \int_{\Omega} \frac{1}{|x-y|} d x d y-\operatorname{Per}\left(\Omega^{*}\right) \\
&-\frac{1}{2} \int_{\Omega^{*}} \int_{\Omega^{*}} \frac{1}{|x-y|} d x d y
\end{aligned}
$$

where $\Omega^{*}$ is the ball centered at 0 with volume $\left|\Omega^{*}\right|=|\Omega|=m$. We need to prove that if $m$ is small, then $D(\Omega)>0$ unless $\Omega$ is a ball. Denote

$$
f=\chi_{\Omega^{*}}-\chi_{\Omega}, \quad V=f *|x|^{-1} .
$$

By a quantitative isoperimetric inequality in [8], there exists a universal constant $\epsilon_{0}>0$ such that after an appropriate translation of $\Omega$, we have

$$
\operatorname{Per}(\Omega)-\operatorname{Per}\left(\Omega^{*}\right) \geqslant \epsilon_{0} \int_{\mathbb{R}^{3}} \frac{f(x)}{|x|} d x=\epsilon_{0} V(0) .
$$

Note that by Hardy-Littlewood rearrangement inequality, $V(0)>0$ unless $\Omega$ is a ball. For the Coulomb terms, we can write

$$
\begin{aligned}
\frac{1}{2} & \int_{\Omega} \int_{\Omega} \frac{1}{|x-y|} d x d y-\frac{1}{2} \int_{\Omega^{*}} \int_{\Omega^{*}} \frac{1}{|x-y|} d x d y \\
& =\frac{1}{2} \int_{\mathbb{R}^{3}} \int_{\mathbb{R}^{3}} \frac{f(x) f(y)}{|x-y|} d x d y+\int_{\mathbb{R}^{3}} \int_{\mathbb{R}^{3}} \frac{\chi_{\Omega^{*}}(x) f(y)}{|x-y|} d x d y \\
& =\frac{1}{8 \pi} \int_{\mathbb{R}^{3}}|\nabla V(x)|^{2} d x+\int_{\Omega^{*}} V(x) d x .
\end{aligned}
$$

In the last equality we used $-\Delta V=4 \pi f$. This Poison equation also shows that $V$ is superharmonic in $\Omega^{*}$ (as $f \geqslant 0$ in $\Omega^{*}$ ), and hence

$\int_{\mathbb{R}^{3}} \int_{\mathbb{R}^{3}} \frac{\chi_{\Omega^{*}}(x) f(y)}{|x-y|} d x d y=\int_{\Omega^{*}} V(x) d x \leqslant\left|\Omega^{*}\right| V(0)=m V(0)$.

Thus in summary, if $m<\epsilon_{0}$ and $\Omega$ is not a ball, then

$$
D(\Omega) \geqslant\left(\epsilon_{0}-m\right) V(0)>0 \text {. }
$$

Nonexistence if $m>8$ [5].

Assume that $E^{\mathrm{G}}(m)$ has a minimizer $\Omega$. We split $\Omega$ into two parts, $\Omega=\Omega^{+} \cup \Omega^{-}$, by a hyperplane $H$ and then move $\Omega^{-}$to infinity by translations. Since $\Omega$ is a minimizer, we obtain

$$
\begin{aligned}
\operatorname{Per}(\Omega)+\int_{\Omega} \int_{\Omega} \frac{1}{|x-y|} d x d y & \\
\leqslant & \operatorname{Per}\left(\Omega^{+}\right)+\int_{\Omega^{+}} \int_{\Omega^{+}} \frac{1}{|x-y|} d x d y \\
& +\operatorname{Per}\left(\Omega^{-}\right)+\int_{\Omega^{-}} \int_{\Omega^{-}} \frac{1}{|x-y|} d x d y
\end{aligned}
$$

which is equivalent to

$$
2 \mathcal{H}^{2}(\Omega \cap H) \geqslant \int_{\Omega^{+}} \int_{\Omega^{-}} \frac{1}{|x-y|} d x d y .
$$

Here $\mathcal{H}^{2}$ is the two-dimensional Hausdorff measure. Next, we parameterise:

$$
H=H_{v, \ell}=\left\{x \in \mathbb{R}^{3}: x \cdot v=\ell\right\}
$$

with $v \in S^{2}, \ell \in \mathbb{R}$. The above inequality becomes

$$
2 \mathcal{H}^{2}\left(\Omega \cap H_{v, \ell}\right) \geqslant \int_{\Omega} \int_{\Omega} \frac{\chi(v \cdot x>\ell>v \cdot y)}{|x-y|} d x d y .
$$

Integrating over $\ell \in \mathbb{R}$ and using Fubini's theorem we get

$$
2|\Omega| \geqslant \int_{\Omega} \int_{\Omega} \frac{[v \cdot(x-y)]_{+}}{|x-y|} d x d y .
$$

Finally, averaging over $v \in S^{2}$ and using

$$
\int[v \cdot z]_{+} \frac{d v}{4 \pi}=\frac{|z|}{2} \int_{0}^{\pi / 2} \cos \theta \sin \theta d \theta=\frac{|z|}{4}
$$

with $z=(x-y)$, we conclude that $2|\Omega| \geqslant \frac{1}{4}|\Omega|^{2}$, namely $|\Omega| \leqslant 8$.

With Rupert Frank and Hanne Van Den Bosch, we used the cutting argument in the liquid drop model to study the ionization problem in the Thomas-Fermi-Dirac-von Weisäcker theory in [6], and in the Müller density matrix functional theory in [7]. In these theories, the standard Benguria-Lieb method does not apply, but we can replace it by an appropriate modification of the minimizers, leading to an efficient control of the number of particles "outside" in terms of particles "inside". This enables us to employ Solovej's bootstrap argument to establish the uniform bound $N-Z \leqslant C$.

\section{$6 \quad$ Related problems}

The ionization problem is an example for a question that is easy to find in physics textbooks, but difficult to answer mathematically. Below we list some related open problems for the Schrödinger operator $H_{N}$.

The main concept in the ionization problem is that in a large atom, although most of electrons stay in the domain $|x| \sim$ $Z^{-1 / 3}$, the binding property only depends on a few outermost electrons in the region $|x| \sim 1$. In fact, only this outer region is relevant to chemical reactions in everyday life. Therefore, an important quantity of an atom is its radius. To fix the notation, we define the radius $R_{\Psi}$ of a wave function $\Psi$ by requiring

$$
\int_{|x| \geqslant R_{\Psi}} \rho_{\Psi}(x) d x=1 .
$$

Conjecture 11 ([24]). There exist two universal constants $0<R_{1}<R_{2}$ such that if $N \geqslant Z$ and $E_{N}$ has a minimizer $\Psi$, then $R_{1} \leqslant R_{\Psi} \leqslant R_{2}$.

Another important quantity is the ionization energy $I_{N}=$ $E_{N-1}-E_{N}$.

Conjecture 12 ([15,23]). There exists a universal constant $C>0$ such that if $N \geqslant Z$, then $I_{N} \leqslant C$.

Conjecture 13 ([15]). The function $N \mapsto I_{N}$ is non-increasing (equivalently $N \mapsto E_{N}$ is convex).

See [20] for partial results on Conjectures 11 and 12. A consequence of Conjecture 13 is that if $E_{N-1}>E_{N}$ (namely the nucleus can bind $N$ electrons), then $E_{N-2}>E_{N-1}$ (the nucleus can bind $N-1$ electrons). This "obvious fact" is still not proved mathematically!

So far we have only focused on the ground state problem for $H_{N}$. Recall from the HVZ theorem that the essential spectrum of $H_{N}$ is $\left[E_{N-1}, \infty\right)$. Conjecture 2 mainly concerns the existence of eigenvalues below $E_{N-1}$. Since the existence of embedded eigenvalues is generally not expected, we have the following stronger version of Conjecture 2.

Conjecture 14. There exists a universal constant $C>0$ such that if $N>Z+C$, then $H_{N}$ has no eigenvalue.

The last issue has been studied by Lenzmann and Lewin in [11], who proved that $H_{N}$ has no eigenvalue if $N>4 Z+1$. 
This question is related to the scattering theory of dispersive PDEs with long-range interaction potentials, which is interesting in its own right.

\section{Bibliography}

[1] R. Benguria and E. H. Lieb, Proof of stability of highly negative ions in the absence of the Pauli principle. Phys. Rev. Lett. 50 (1983), 1771-1774.

[2] R. Choksi and M. A. Peletier, Small volume-fraction limit of the diblock copolymer problem: II. Diffuse-interface functional. SIAM J. Math. Anal. 43 (2011), 739-763.

[3] R. Choksi, C. B. Muratov, I. Topaloglu, An Old Problem Resurfaces Nonlocally: Gamow's Liquid Drops Inspire Today's Research and Applications. Notices of the AMS, December 2017.

[4] C. Fefferman and L. A. Seco, Asymptotic neutrality of large ions, Commun. Math. Phys. 128(1990), 109-130.

[5] R. L. Frank, R, Killip, P. T. Nam, Nonexistence of large nuclei in the liquid drop model. Lett. Math. Phys. 106 (2016), 10331036.

[6] R. L. Frank, P. T. Nam and H. Van Den Bosch, The ionization conjecture in Thomas-Fermi-Dirac-von Weizsäcker theory. Comm. Pure Appl. Math. 71 (2018), 577-614.

[7] R. L. Frank, P. T. Nam and H. Van Den Bosch, The maximal excess charge in Müller density-matrix-functional theory. Ann. Henri Poincaré 19 (2018), 2839-2867.

[8] N. Fusco and V. Julin, A strong form of the Quantitative Isoperimetric inequality. Calc. Var. PDE 50 (2014), 925-937.

[9] H. Knüpfer, C. B. Muratov. On an isoperimetric problem with a competing nonlocal term II: The general case. Comm. Pure Appl. Math. 67 (2014), pp. 1974-1994.

[10] V. Julin, Isoperimetric problem with a Coulombic repulsive term. Indiana Univ. Math. J. 63 (2014), 77- 89.

[11] E. Lenzmann and M. Lewin, Dynamical Ionization Bounds for Atoms. Anal. PDE 6 (2013), 1183-1211.

[12] E. H. Lieb, Bound on the maximum negative ionization of atoms and molecules. Phys. Rev. A 29 (1984), 3018-3028.

[13] E. H. Lieb, I. M. Sigal, B. Simon, and W. Thirring, Asymptotic Neutrality of Large-Z Ions. Phys. Rev. Lett. 52 (1984), 994 996.
[14] E. H. Lieb and B. Simon, The Thomas-Fermi theory of atoms, molecules and solids. Adv. Math. 23 (1977), 22-116.

[15] E. H. Lieb and R. Seiringer, The stability of matter in quantum mechanics, Cambridge University Press, 2009.

[16] J. Lu and F. Otto, Nonexistence of a Minimizer for ThomasFermi-Dirac-von Weizsäcker Model. Comm. Pure Appl. Math. 67 (2014), 1605-1617.

[17] P. T. Nam, New bounds on the maximum ionization of atoms. Commun. Math. Phys. 312 (2012), 427-445.

[18] P. T. Nam, On the number of electrons that a nucleus can bind, Proceedings of the ICMP 2012.

[19] M. B. Ruskai, Absence of discrete spectrum in highly negative ions, II. Extension to Fermions. Commun. Math. Phys. 82 (1982), 325-327.

[20] L. A. Seco, I. M. Sigal, and J. P. Solovej, Bound on the ionization energy of large atoms. Commun. Math. Phys. 131 (1990), 307-315.

[21] I. M. Sigal, Geometric methods in the quantum many-body problem. Nonexistence of very negative ions, Commun. Math. Phys. 85 (1982), 309-324.

[22] I. M. Sigal, How many electrons can a nucleus bind? Ann. Phys. 157 (1984), 307-320.

[23] B. Simon, Schrödinger operators in the twenty-first century. In Mathematical Physics 2000, Imperial College Press, 283-288.

[24] J. P. Solovej, The ionization conjecture in Hartree-Fock theory. Ann. of Math. 158 (2003), 509-576.

[25] J.P. Solovej, A new look at Thomas-Fermi theory. Molecular Physics 114 (2016), 1036-1040.

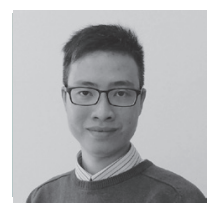

Phan Thành Nam [nam@math.lmu.de] is a mathematical physicist working on analytical problems from quantum physics. He is particularly interested in the spectral theory of many-body quantum systems, including atoms, molecules and Bose gases. He is currently a professor of mathematics at LMU Munich. 